\title{
World Wide Wisdom Web (W4) and Autonomy Oriented Computing (AOC): What, When, and How? \\ (Invited Talk)
}

\author{
Jiming Liu \\ Department of Computer Science, \\ Hong Kong Baptist University, \\ Kowloon Tong, Hong Kong \\ jiming@comp.hkbu.edu.hk
}

The World Wide Wisdom Web (W4) (or Wisdom Web) and Autonomy Oriented Computing (AOC) were proposed and advocated by Liu et al. and have been recognized, in the fields of WWW, AI, and AAMAS, as promising directions of research in problems that are heavily dominated by large scale, distribution, connectivity, interactivity, and redundancy [1/7/8/2]4.

Generally speaking, W4 encompasses the systems, environments, and activities (1) that are empowered through the global or regional connectivity of computing resources as well as distribution of data, knowledge, and contextual presence, and (2) that are specifically dedicated to enable human beings to gain practical wisdoms of working, living, and playing throughout their professional and personal activities, such as running a business and living with a certain lifestyle. W4 will become the next generation Web Intelligence (WI), providing not only a medium for seamless knowledge and experience sharing but also a supply of self-organized resources for driving sustainable knowledge creation and scientific or social development/evolution.

The present challenges of W4 lie primarily in:

1. how to autonomously derive just-in-time, optimal or sub-optimal solutions to an application-dependent problem, and delegate/carry out related tasks in a distributed way;

2. how to dynamically discover and mobilize distributed, constantly changing (hence sometimes unreliable), redundant or incomplete, heterogeneous resources that can exist in the forms of computational utilities, data repositories, and knowledge sources;

3. how to support the emergence of new social interactions and norms, and cultivate collective and social intelligence on the basis of (1) and (2) above.

The above problems have been known as the three benchmarks or dimensions for operationally defining and evaluating $\mathrm{W} 41$.

In $\mathrm{W} 4$, the tasks of computing are seamlessly carried out through a variety of agent embodiments. There is no single multi-purpose or dedicated machine 
that can manage to accomplish a job of this nature. The key to success in such an application lies in a large-scale deployment of Wisdom agents capable of autonomously performing localized interactions and making rational decisions in order to achieve their collective goals [5]. In other words, W4 readily exploits and explores the new paradigm of Autonomy Oriented Computing (AOC) 214.

In essence, the AOC paradigm is intended to effectively and efficiently achieve emergent computational autonomy that is useful in tackling hard computational problems, such as those that are:

1. of high-complexity - problems that involve a large number of autonomous entities, large-scale, high-dimension, highly nonlinear interactions or relationships, and highly interrelated/constrained variables;

2. highly-distributed and locally-interacting - problems that are not solvable in a centralized manner nor ready/efficient for batch processing.

AOC is a self-organizing computing paradigm, which has been found appealing in the areas of large-scale computation, distributed constraint satisfaction, decentralized optimization, and complex phenomena or emergent behavior characterization [35]6]4].

This talk presents the theoretical and experimental work on W4, as carried out in the AAMAS/AOC lab of HKBU, and highlights the methodologies of AOC in terms of formulation, macroscopic modeling, discrete-event simulations, and empirical studies of dynamics.

\section{Acknowledgements}

I am grateful to my research collaborators, research assistants, and students who have, over the years, worked together with me on the development of Web Intelligence (WI), World Wide Wisdom Web (W4), and Autonomy Oriented Computing (AOC). I would like to acknowledge the support of Hong Kong Research Grant Council (RGC) Central Allocation Grant ("Web Intelligence for E-Transformation - A Research Centre" - HKBU 2/03C).

\section{References}

1. Liu, J. (2003). Web Intelligence (WI): What makes Wisdom Web? Proceedings of the Eighteenth International Joint Conference on Artificial Intelligence (IJCAI-03), Acapulco, Mexico, Aug. 9-15, 2003, Morgan Kaufmann Publishers, pp. 1596-1601 (invited talk).

2. Liu, J. (2001). Autonomous Agents and Multi-Agent Systems: Explorations in Learning, Self-Organization and Adaptive Computation, World Scientific.

3. Liu, J., Han, J., and Tang, Y. Y. (2002). Multi-agent oriented constraint satisfaction, Artificial Intelligence, 136, 1, pp. 101-144.

4. Liu, J., Jin, X., and Tsui, K.C. (2005a). Autonomy Oriented Computing (AOC): From Problem Solving to Complex Systems Modeling, Springer. 
5. Liu, J., Jin, X., and Wang, S. (2005b). Agent-based load balancing on homogeneous minigrids: Macroscopic modeling and characterization, IEEE Transactions on Parallel and Distributed Systems, 16, 6.

6. Liu, J., Zhang, S., and Yang, J. (2004). Characterizing Web usage regularities with information foraging agents, IEEE Transactions on Knowledge and Data Engineering, 16, 5, pp. 566-584.

7. Liu, J., Zhong, N., Yao, Y. Y., and Ras, Z. W. (2003). The Wisdom Web: New challenges for Web Intelligence (WI), Journal of Intelligent Information Systems, Kluwer Academic Publishers, 20, 1.

8. Zhong, N., Liu, J., and Yao, Y. Y. (2003). (Eds.), Web Intelligence, Springer. 